\title{
Mode of action of Jatropha curcas phorbol esters in bovine kidney cells
}

\begin{abstract}
Jatropha meal is a potential biofeed for animal production however, the presence of phorbol esters (PEs) in the meal limits its utilization. The PEs caused severe toxic manifestations in animals. The information on the mechanisms of toxicity at the cellular level is rather limited. Therefore, this research was conducted to investigate the mode of action of PEs by evaluating the biochemical, biological and molecular responses of cells exposed to PEs. Phorbol esters from Jatropha meal were isolated and identified as Jatropha factor (JF) JFC1, JFC2, JFC3 and a mixture of JFs (C4, C5 and C6). These PEs exhibited cytotoxic activity towards bovine kidney cells and induced significant morphological changes. The PEs severely altered the redox status of the cells which resulted in the occurrence of oxidative stress. Moreover, the PEs upregulated the expressions of PKC- $\beta \mathrm{II}$, proto-oncogenes, pro-inflammatory cytokines and elevated the caspase- 3 activity in the cells. All PEs were toxic, but JFC1 and JFC2 appeared to be more toxic to the bovine kidney cells as compared to the JFC3 and the mixture of JFs. The results indicated that the cytotoxic activity of Jatropha meal PEs was elicited through induction of oxidative stress and inflammation of the cells.
\end{abstract}

Keyword: Toxicity; Molecular mechanism; Inflammation; Apoptosis; Animal feed; Biological activity 